\title{
Clinical Study \\ Usefulness of the Core Outcome Measures Index in Daily Clinical Practice for Assessing Patients with Degenerative Lumbar Disease
}

\author{
Carlos Lozano-Álvarez, Daniel Pérez-Prieto, Guillem Saló, Antoni Molina, \\ Andreu Lladó, and Manuel Ramírez
}

Department of Orthopaedic Surgery and Traumatology, Parc de Salut Mar, Hospitales del Mar y la Esperanza, 08003 Barcelona, Spain

Correspondence should be addressed to Carlos Lozano-Álvarez, 97486@parcdesalutmar.cat

Received 28 October 2011; Revised 17 December 2011; Accepted 20 December 2011

Academic Editor: Brian R. Subach

Copyright (C) 2012 Carlos Lozano-Álvarez et al. This is an open access article distributed under the Creative Commons Attribution License, which permits unrestricted use, distribution, and reproduction in any medium, provided the original work is properly cited.

\begin{abstract}
Introduction. Outcome evaluation is an important aspect of the treatment of patients with degenerative lumbar disease. We evaluated the usefulness of the Core Outcome Measures Index (COMI) in assessing people affected by degenerative lumbar disease in daily clinical practice. Methods. We evaluated 221 patients who had completed preoperatively and 2 years after surgery VAS pain, Short Form-36 (SF-36), Oswestry Disability Index (ODI) and COMI. We calculated the change of scores and its sensitivity to change. The internal consistency of the COMI items and the correlation between the COMI scores and the scores of the other measurements were assessed. Results. Statistically significant differences were observed between the mean scores of the preoperative and 2 years questionnaires for nearly all measurements. COMI showed a good internal consistency, except for the preoperative pain subscale. The sensitivity to change was high for the total COMI and its pain and well-being subscales and moderate for the rest. The COMI demonstrated strong correlation with the other measurements. Conclusions. The COMI is a useful tool for assessing the patient-based outcome in the studied population. Given its simplicity, good correlation with the SF-36 and ODI and its good sensitivity to change, it could replace more cumbersome instruments in daily clinical practice.
\end{abstract}

\section{Introduction}

Degenerative lumbar disease (DLD) and chronic low back pain (CLBP) are orthopaedic problems of the highest incidence in the Spanish population [1]. In the United States, the lifetime prevalence of low back pain has been reported to be as high as $84 \%$ and the prevalence of CLBP to be about $23 \%$, with $11-12 \%$ of the population being disabled by low back pain [2]. Often, DLD and CLBP require surgical intervention so that DLD has become the leading cause of arthrodesis in the spine [1]. In the USA, the annual number of lumbar fusions for degenerative lumbar disease has increased from 174,223 in 1998 to 413,171 in 2008 [3].

Patient-based outcomes may be the most important tool clinicians, patients, and policymakers can use to identify the effectiveness of different low back pain treatments. In 1998, a multinational group of back pain investigators designed the Core Outcome Measures Index (COMI) to evaluate pain, function, generic health status or well-being, disability, and satisfaction [4]. The COMI ultimate goal was to provide a standardized outcome assessment without an excessive burden of instruments or questions that make it difficult for patients to complete the instruments of evaluation. The COMI was validated against well-validated instruments such as the Roland-Morris or the Oswestry Disability Index (ODI) for back specific function and the Medical Outcomes Study Short Form-36 (SF-36), its Short Form SF-12, or the EuroQol for general health status. In 2006, a Spanish group validated the Spanish version of the COMI [5]. The authors designed a prospective study that aimed to evaluate the reliability, validity, and responsiveness of this instrument. They evaluated this instrument in patients with subacute osteoporotic fracture (quick improvement of the pain after treatment) and chronic low back pain (slow improvement of the pain) and related the COMI scores to the scores of the Spanish-validated ODI, SF-36, and SF-12. They concluded 
that the COMI was a useful tool to evaluate patient-based outcomes when the respondent burden is an important problem. Still, subscale scores needed to be further tested in other populations.

The objective of our study is to evaluate the usefulness of the COMI as an outcome measurement in daily clinical practice for patient suffering from DLD.

\section{Material and Methods}

2.1. Patient Sample. We reviewed the outcomes from 263 patients operated between 2005 and 2008 for degenerative lumbar disease. Of those 263 patients who had completed the preoperative questionnaires, 221 also completed the questionnaires 2 years after surgery. Thirty-five of the 42 patients without postoperative outcomes could not be found, and 7 had died.

Patients were excluded if they were younger than 18 years old, had surgeries for infectious disease, tumours, or rheumatic origin, or had a language barrier that prevented them from properly understanding the questionnaires. We included all patients older than 18 years old who were operated for the following diagnoses: degenerative disc disease, stenosis, disc herniation, spondylolisthesis, and pseudarthrosis. Epidemiological data collected during the study were age, sex, employment status, diagnosis, surgical procedure, and degree of comorbidity on the American Society of Anaesthesiologists (ASA) scale [6, 7].

2.2. Questionnaires. All patients were clinically evaluated and then self-completed the validated Spanish version of the SF-36 version $2[8,9]$ to evaluate their general health, the validated Spanish version of the ODI $[10,11]$ to assess their disability, visual analogue scales (VAS) $[12,13]$ to evaluate lumbar and sciatic pain, and the validated Spanish version of the COMI $[4,5]$ used to comprehensively evaluate patients. All questionnaires were filled out before surgery and 2 years after surgery.

The COMI $[4,14]$ is a questionnaire composed of 8 questions that evaluates pain ( 2 items), function ( 1 item), well-being (1 item), disability (2 items) and satisfaction (2 items). The scores of the questionnaire range from 1 to 5 , with 1 being the best possible result. The total COMI score is the average of the 5 dimensions. It was designed for a simpler but effective standardized evaluation of outcome in patients with low back pain and would replace more cumbersome health-related questionnaires in daily practice.

2.3. Data Analysis. Statistical analyses were performed with SPSS 15.0 (SPSS Inc., Chicago, IL, USA). Student's $t$-tests were used to compare the pre-operative and post-operative scores; a $P$ value below 0.05 was considered statistically significant. The change in scores from pre-operative to 2year follow-up was calculated as the preoperative scores minus the post-operative scores. A negative change score indicates improvement for ODI, COMI, and VAS, while a positive change score indicates improvement for the SF36. The magnitude of change (sensitivity to change) was assessed by the standardized mean response (SMR). SMR is
Table 1: Epidemiological data (221 patients).

\begin{tabular}{lc}
\hline Age years & $($ range $)$ \\
55.1 & $(22-86)$ \\
\hline Sex & $n(\%)$ \\
Female & $112(50,7)$ \\
Male & $109(49,3)$ \\
\hline Employment situation & $n(\%)$ \\
Employed & $119(53,8)$ \\
Permanent disability & $27(12,2)$ \\
Temporary disability & $28(12.7)$ \\
Retired & $40(18,1)$ \\
Unemployed & $7(3,2)$ \\
\hline ASA score & $($ range $)$ \\
2 & $(1-4)$ \\
\hline Diagnostics & $n(\%)$ \\
DDD & $86(38,9)$ \\
Lumbar stenosis & $76(34,3)$ \\
Disc herniation & $28(12,7)$ \\
Spondylolisthesis & $16(7,2)$ \\
Pseudarthrosis & $15(6,7)$ \\
\hline Treatment & $n(\%)$ \\
TLIF & $80(36,2)$ \\
Posterolateral fusion & $71(32,1)$ \\
PLIF & $31(14,03)$ \\
Discectomy & $28(12,7)$ \\
Laminectomy & $11(4,97)$ \\
\hline
\end{tabular}

one of the possible calculations of effect size; specifically it is obtained by dividing the mean difference by the standard deviation of the change scores [15]. The use of effect size allows for comparisons between different outcome measures because it translates score differences into a standard unit of measurement. Applying Cohen's threshold values of effect size to SMR, sensitivity is considered trivial for SMR values lower than 0.20, small for SMR values between 0.20 and 0.50, moderate for SMR values between 0.50 and 0.80 , and large for SMR values greater than 0.80 [16].

The internal consistency of the various questionnaires was evaluated with Cronbach's alpha test. Cronbach's alpha was not applicable for the function and well-being subscales of the COMI because they are composed of a single item. Alpha values between 0.8 and 0.9 indicate a good consistency, and values between 0.7 and 0.8 indicate an acceptable level of consistency [17].

Construct validity was assessed through Pearson's correlation. Items measuring similar concepts were expected to have high correlation coefficients $(>0.6)$, and items measuring different concepts were expected to have low correlation coefficients $(<0.4)$ [5].

\section{Results}

The mean patient age was 55.1 years (22 to 86 years), and 112 patients $(50.7 \%)$ were women. At the time of surgery, $53.8 \%$ 
TABLE 2: Preoperative and 2-year postoperative scores and magnitude of change.

\begin{tabular}{|c|c|c|c|c|c|c|c|}
\hline \multirow{2}{*}{ Health status measures } & \multicolumn{2}{|c|}{ Preoperative } & \multicolumn{2}{|c|}{ Postoperative } & \multicolumn{2}{|c|}{ Difference } & \multirow{2}{*}{ SMR } \\
\hline & Mean & $\mathrm{SD}$ & Mean & $\mathrm{SD}$ & Mean & $\mathrm{SD}$ & \\
\hline ODI & 45.60 & 17.85 & 36.60 & 22.24 & $-8.76^{*}$ & 19.43 & -0.451 \\
\hline COMI & 3,77 & 0.76 & 2.60 & 0.53 & $-1.07^{*}$ & 1.19 & -0.899 \\
\hline Pain & 3.88 & 0.89 & 2.79 & 1.27 & $-1.13^{*}$ & 1.28 & -0.883 \\
\hline Function & 3.99 & 1.12 & 2.90 & 1.53 & $-1.01^{*}$ & 1.68 & -0.601 \\
\hline Well-being & 4.83 & 0.47 & 3.19 & 1.49 & $-1.60^{*}$ & 1.48 & -1.081 \\
\hline Disability & 3.35 & 1.67 & 1.89 & 1.29 & $-1.01^{*}$ & 1.85 & -0.546 \\
\hline Satisfaction & 2.81 & 1.09 & 2.21 & 1.34 & $-0.61^{*}$ & 1.15 & -0.530 \\
\hline \multicolumn{8}{|l|}{ SF36v2 } \\
\hline Physical Function (PF) & 29.29 & 9.48 & 36.68 & 12.79 & $7.18^{*}$ & 12.05 & 0.596 \\
\hline Role physical (RP) & 30.66 & 8.76 & 21.17 & 4.37 & $-9.79^{*}$ & 9.46 & -1.035 \\
\hline Bodily pain (BP) & 30.25 & 6.99 & 39.10 & 12.56 & $8.87^{*}$ & 12.54 & 0.707 \\
\hline General health $(\mathrm{GH})$ & 42.16 & 9.01 & 39.83 & 11.98 & $-1.90^{* *}$ & 11.13 & -0.171 \\
\hline Vitality (VT) & 35.35 & 9.36 & 44.17 & 12.01 & $8.82^{*}$ & 11.69 & 0.755 \\
\hline Social function (SF) & 30.45 & 13.71 & 39.68 & 13.89 & $8.56^{*}$ & 15.71 & 0.545 \\
\hline Role emotional (RE) & 36.26 & 14.82 & 16.10 & 5.39 & $-20.38^{*}$ & 13.91 & -1.465 \\
\hline Mental health (MH) & 39.40 & 10.64 & 37.90 & 11.92 & -1.09 & 1.77 & -0.085 \\
\hline PCS & 30.90 & 7.41 & 36.66 & 10.89 & $6.38^{*}$ & 10.9 & 0.585 \\
\hline MCS & 39.91 & 12.28 & 32.78 & 9.76 & $-6.10^{*}$ & 12.50 & -0.488 \\
\hline \multicolumn{8}{|l|}{ VAS } \\
\hline Back & 7.55 & 2.15 & 5.40 & 3.41 & $2.01^{*}$ & 3.34 & 0.601 \\
\hline Sciatica & 7.62 & 2.62 & 4.18 & 3.47 & $2.39 *$ & 3.81 & -0.627 \\
\hline
\end{tabular}

${ }^{*} P<0.001,{ }^{* *} P<0.05$.

Sensitivity to change (SMR): low $\approx 0.2$; moderate $\approx 0.5$. High $>0.8$.

of the patients were employed, $24.9 \%$ were on disability, $18.1 \%$ were retired, and $3.2 \%$ were unemployed (Table 1 ).

The most frequent causes of surgical intervention were degenerative disc disease (DDD, 38.9\% of cases) and lumbar spinal stenosis $(34.3 \%)$. The most common surgical treatments were transforaminal lumbar interbody fusion (TLIF, $36.2 \%)$ and posterolateral fusion (32.1\%). The average degree of comorbidity measured by ASA scale was 2 with a range from 1 to 4 (Table 1).

3.1. Magnitude of Change and Responsiveness. There was an overall improvement in the average scores of the different questionnaires from the preoperative visit to the visit at 2 years, and this difference was statistically significant in all measures except for the mental health subscale of the SF-36 (Table 2). The SMR indicated a high sensitivity to change for the total COMI and its pain and well-being subscales. The sensitivity to change was moderate for the COMI function, disability, and satisfaction subscales (Table 2).

3.2. Internal Consistency. Cronbach's alpha indicated a good internal consistency of the COMI both in the preoperative phase $($ alpha $=0.807)$ and the 2-year evaluation (alpha $=$ 0.91 ), except for the preoperative pain subscale (Table 3 ). The low internal consistency of the pain subscale may be due to the fact that it is a combination of back pain and leg pain
TABLE 3: Internal Consistency (cronbach's alpha coefficient).

\begin{tabular}{lcc}
\hline & & $\alpha$ \\
ODI & Preoperative & Postoperative \\
COMI (Total) & 0.854 & 0.915 \\
Pain & 0.807 & 0.910 \\
Function & 0.446 & 0.776 \\
Well-being & - & - \\
Disability & - & - \\
Satisfaction & 0.911 & 0.749 \\
SF-36v2 & 0.827 & 0.858 \\
PF & & \\
GH & 0.888 & 0.938 \\
RP & 0.750 & 0.839 \\
RE & 0.889 & 0.938 \\
SF & 0.879 & 0.932 \\
BP & 0.662 & 0.856 \\
VT & 0.768 & 0.887 \\
MH & 0.828 & 0.811 \\
\hline
\end{tabular}

$\alpha>0.7-0.8($ max value $=1) \rightarrow$ good reliability.

while the patients in our sample were not likely to evenly suffer from back and leg pain. 
TABle 4: Pearson's correlation coefficients.

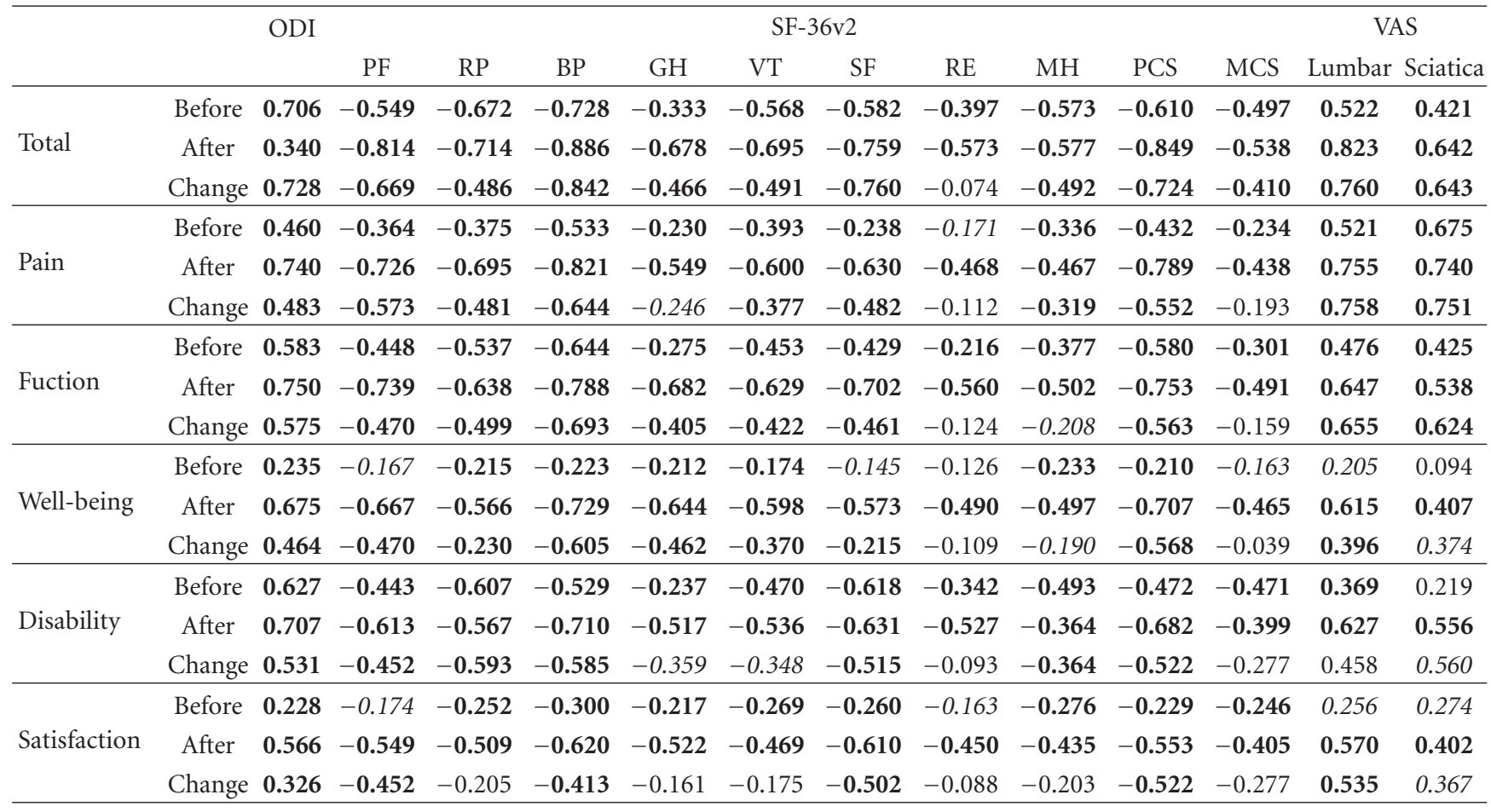

Statistical significance $(P): P<0.05$ (italics). $P<0.01$ (bold).

3.3. Construct Validity. Pearson's correlation coefficients indicated that the COMI total score and its subscales had a statistically significant correlation with almost all values of the ODI, SF-36, and VAS before surgery and after surgery and the score difference (Table 4). In general, items measuring similar concepts had a high $(>0.6)$ correlation coefficient, for instance, the total COMI and ODI $(r=0.7)$ or the disability scale of the COMI and ODI $(r=0.6)$. Items measuring different concepts had low $(<0.4)$ correlation coefficients, for example, the COMI satisfaction scale and the PCS of the SF-36 $(r=-0.2)$ or the COMI well-being scale and the PCS $(r=-0.2)$. However, this trend was not consistent for all measures of similar/dissimilar concepts (e.g., $r=$ 0.5 between COMI pain scale and lumbar VAS) and for measurements times (e.g., $r=0.5$ between preoperative COMI pain scale and lumbar VAS while $r=0.8$ between postoperative COMI pain scale and lumbar VAS).

\section{Discussion}

In this study, the COMI demonstrated good internal consistency, validity, and responsiveness to change in our patient population. Its brevity makes it easier for patients to answer. Its simple scoring and free availability simplifies its administration. For all these reasons, the COMI appears a useful measurement tool of patients' outcomes in daily practice.

The COMI was originally designed by a multinational group as a standardized core of questions that assess briefly but globally patients based outcomes [5]. The design took into account factors such as breadth of coverage, demonstrated validity and reproducibility, and demonstrated responsiveness, practicality (brevity and low cost), compatibility with widely promoted instruments or batteries, and importance to patients and society. The resulting COMI is comprised of 5 scales already validated and in use in some form in other instruments such as EuroQol, National Health Interview Survey, the North American Spine Society, and American Academy of Orthopedic Surgeons instruments.

The psychometric characteristics of the COMI were established with a study of the COMI prospectively administered to 277 patients with low back pain. It demonstrated good reliability, reproducibility, validity, and sensitivity of the COMI composite score and subscales [18]. The German [14], French [19], and Italian [20] versions as well as a neck [21] version of the COMI have been validated. It is recommended as "a suitable instrument for implementation in the Spine Tango Registry or in any other multi-language databases of outcomes in LBP patients"... "the systematic and widespread use of this version in similar settings might enhance the quality of the follow-up of patients with chronic LBP" [20]. This instrument is considered in both versions as a practical, reliable, and valid tool and will be of value for international studies and surgical registries.

In 2006, Spanish groups of the Hospital Universitari Vall d'Hebron (Barcelona) and Fundación Jiménez Díaz (Madrid) published the validation study of the Spanish COMI [5]. Their sample included two groups of patients (osteoporotic vertebral fracture and chronic low back pain), and outcomes were evaluated with the Spanish version of COMI and Spanish well-validated versions of SF-36, SF-12, and Oswestry Disability Index. The COMI showed good reproducibility, internal consistency, construct validity, 
and responsiveness, comparable to the more generally used outcome measurements.

The present study examined the use of the COMI in patients with various spine pathologies as typically encountered in daily clinical practice. An important methodological limitation of our study is the lack of test-retest study to confirm the reproducibility of the COMI in our population. Our retrospective analysis did not allow for a test-retest study. Otherwise, just as in the Spanish, German, Italian, and French validation studies, the results of our studies showed similarly good internal consistency, construct validity, and sensitivity to change.

\section{Conclusion}

The COMI is a valid and sensitive questionnaire for the evaluation of patients with degenerative lumbar disease before and after treatment. The results of this study confirm that the COMI is a short, time-saving, easily scored, and multidimensional instrument that can be widely used in daily clinical practice for assessment and monitoring of patients with degenerative lumbar disease.

\section{References}

[1] F. J. Robaina, "Present state of degenerative back surgery and its implications in the management of chronic lumbar pain, canal stenosis and degenerative disk disease. Evidence based outcomes," Revista de la Sociedad Espanola del Dolor, vol. 13, no. 3, pp. 167-172, 2006.

[2] F. Balagué, A. F. Mannion, F. Pellisé, and C. Cedraschi, "Nonspecific low back pain," Lancet, vol. 379, no. 9814, pp. 482-491, 2012.

[3] S. S. Rajaee, H. W. Bae, L. E.A. Kanim, and R. B. Delamarter, "Spinal fusion in the United States: analysis of trends from 1998 to 2008," Spine, vol. 37, no. 1, pp. 67-76, 2012.

[4] R. A. Deyo, M. Battie, A. J. H. M. Beurskens et al., "Outcome measures for low back pain research: a proposal for standardized use," Spine, vol. 23, no. 18, pp. 2003-2013, 1998.

[5] M. Ferrer, F. Pellisé, O. Escudero et al., "Validation of a minimum outcome core set in the evaluation of patients with back pain," Spine, vol. 31, no. 12, pp. 1372-1379, 2006.

[6] A. S. Keats, "The ASA classification of physical status-a recapitulation," Anesthesiology, vol. 49, no. 4, pp. 233-236, 1978.

[7] M. Saklad, "Grading of patients for surgical procedures," Anesthesia, vol. 2, p. 281, 1941.

[8] J. E. Ware Jr. and C. D. Sherbourne, "The MOS 36-item shortform health survey (SF-36)-I. Conceptual framework and item selection," Medical Care, vol. 30, no. 6, pp. 473-483, 1992.

[9] J. Alonso, L. Prieto, and J. M. Antó, "The Spanish version of the SF-36 Health Survey (the SF-36 health questionnaire): an instrument for measuring clinical results," Medicina Clinica, vol. 104, no. 20, pp. 771-776, 1995.

[10] J. C. T. Fairbank and P. B. Pynsent, "The oswestry disability index," Spine, vol. 25, no. 22, pp. 2940-2953, 2000.

[11] S. Alcántara-Bumbiedro, M. T. Flórez-García, C. EchávarriPérez, and F. García-Pérez, "Oswestry low back pain disability questionnaire," Rehabilitacion, vol. 40, no. 3, pp. 150-158, 2006.
[12] E. C. Huskisson, "Measurement of pain," Lancet, vol. 2, no. 7889, pp. 1127-1131, 1974.

[13] M. P. Jensen, C. Chen, and A. M. Brugger, "Postsurgical pain outcome assessment," Pain, vol. 99, no. 1-2, pp. 101-109, 2002.

[14] A. F. Mannion, F. Porchet, F. S. Kleinstück et al., "The quality of spine surgery from the patient's perspective-part 1: the Core Outcome Measures Index in clinical practice," European Spine Journal, vol. 18, no. 3, pp. S367-S373, 2009.

[15] B. Middel and E. van Sonderen, "Statistical significant change versus relevant or important change in (quasi) experimental design: some conceptual and methodological problems in estimating magnitude of intervention-related change in health services research," International Journal of Integrated Care, vol. 2, no. 17, article e15, 2002.

[16] J. Cohen, Statistical Power: Analysis for the Behavioural Sciences, Academic Press, New York, NY, USA, 1977.

[17] L. J. Cronbach, "Coefficient alpha and the internal structure of tests," Psychometrika, vol. 16, no. 3, pp. 297-334, 1951.

[18] A. F. Mannion, A. Elfering, R. Staerkle et al., "Outcome assessment in low back pain: how low can you go?" European Spine Journal, vol. 14, no. 10, pp. 1014-1026, 2005.

[19] S. Genevay, C. Cedraschi, M. Marty et al., "Reliability and validity of the cross-culturally adapted French version of the Core Outcome Measures Index (COMI) in patients with low back pain," European Spine Journal, vol. 21, no. 1, pp. 130-137, 2011.

[20] A. F. Mannion, M. Boneschi, M. Teli et al., "Reliability and validity of the cross-culturally adapted Italian version of the Core Outcome Measures Index," European Spine Journal. In press.

[21] C. D. Fankhauser, U. Mutter, E. Aghayev, and A. F. Mannion, "Validity and responsiveness of the Core Outcome Measures Index (COMI) for the neck," European Spine Journal, vol. 1, no. 1, pp. 101-114, 2011. 


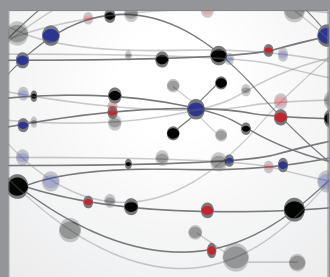

The Scientific World Journal
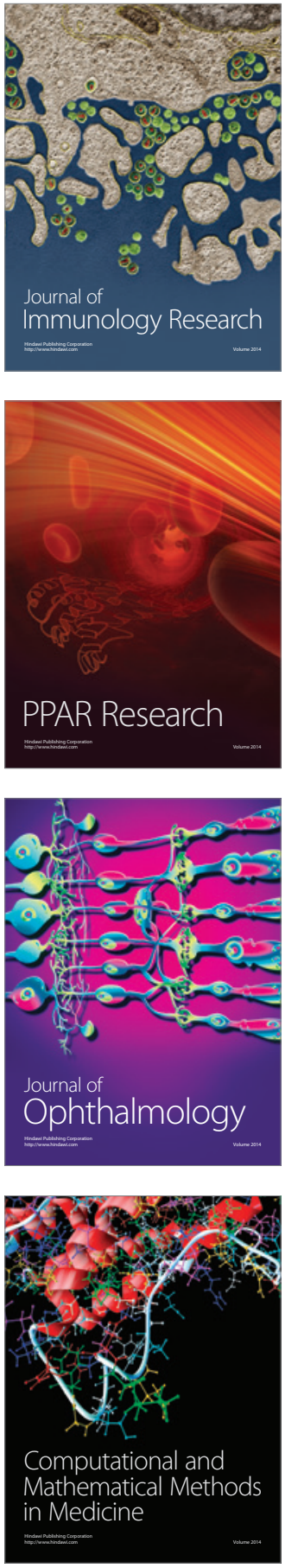

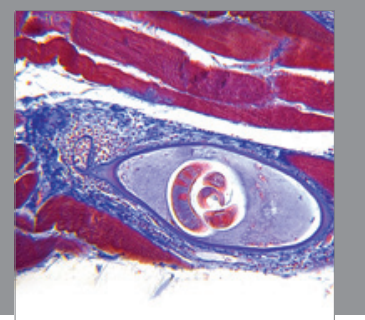

Gastroenterology

Research and Practice
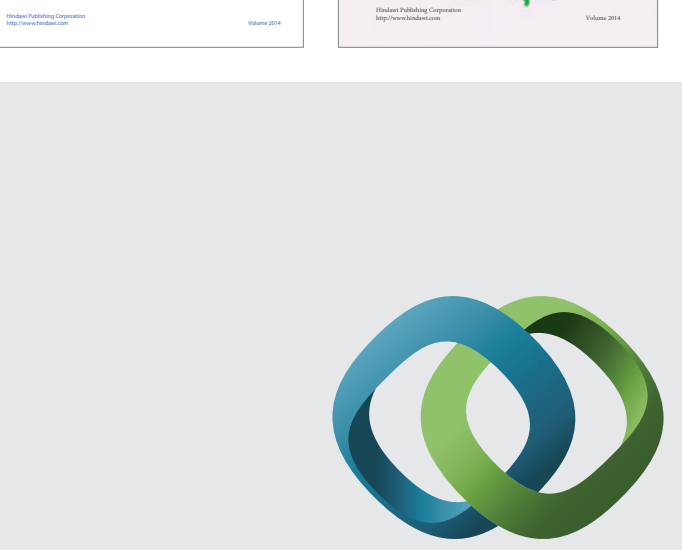

\section{Hindawi}

Submit your manuscripts at

http://www.hindawi.com
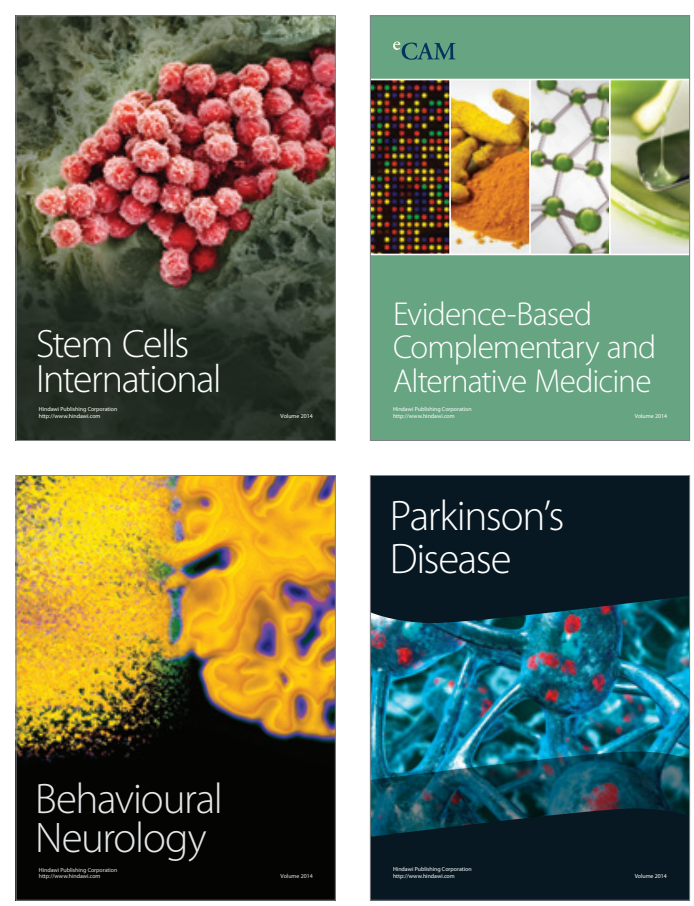

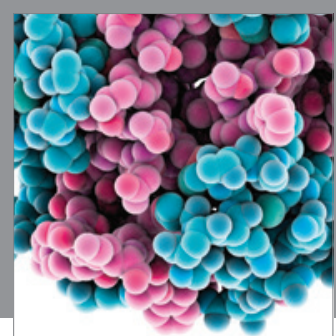

Journal of
Diabetes Research

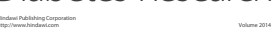

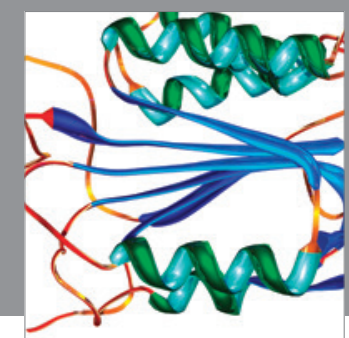

Disease Markers
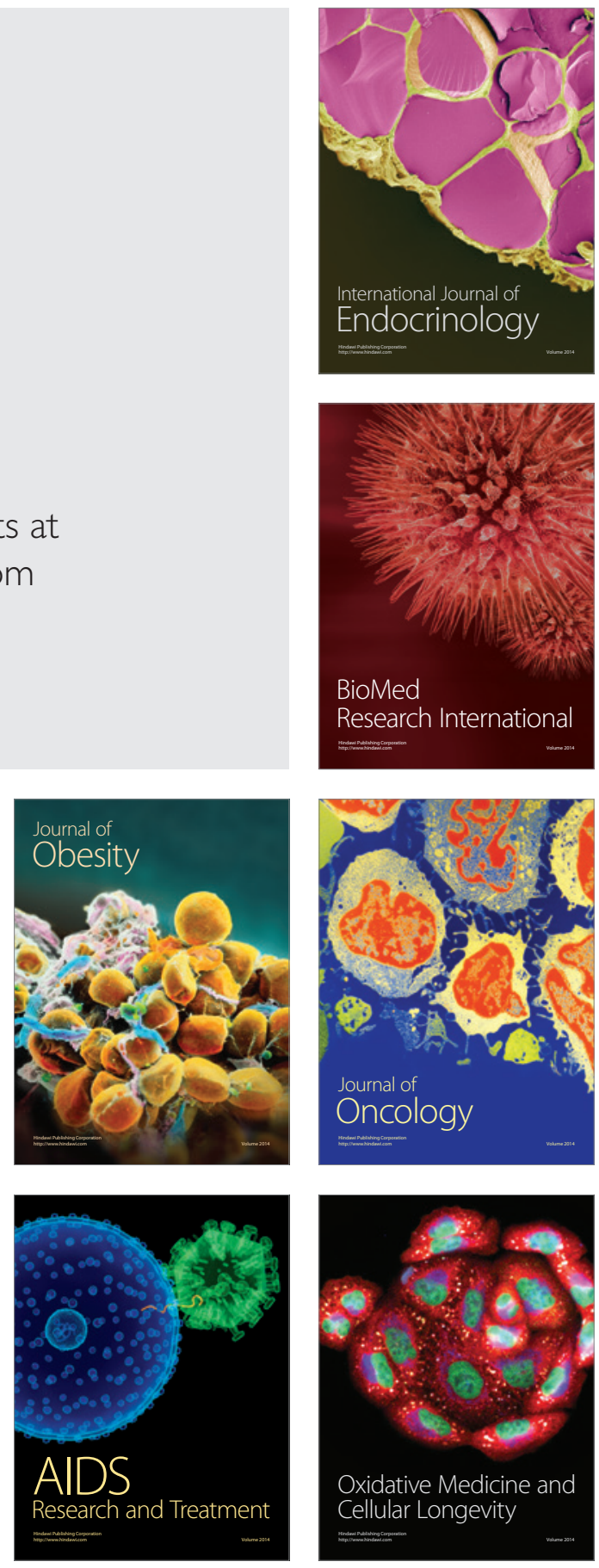